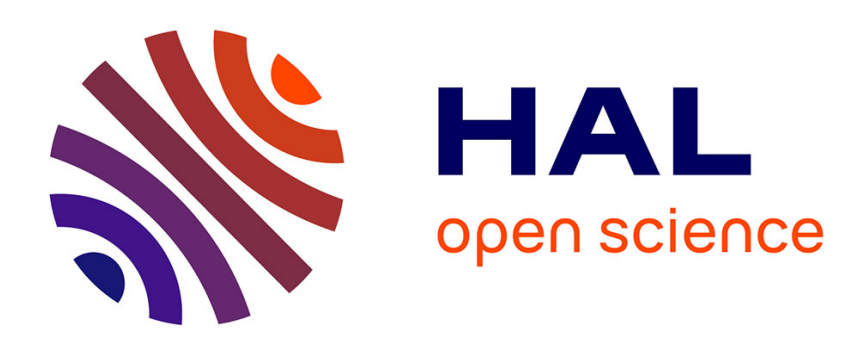

\title{
On the dynamics of the growth plate in primary ossification
}

\author{
A. Fasano, M.A. Herrero, J.M. López, E. Medina
}

\section{To cite this version:}

A. Fasano, M.A. Herrero, J.M. López, E. Medina. On the dynamics of the growth plate in primary ossification. Journal of Theoretical Biology, 2010, 265 (4), pp.543. 10.1016/j.jtbi.2010.05.030 . hal00608942

\section{HAL Id: hal-00608942 \\ https://hal.science/hal-00608942}

Submitted on 16 Jul 2011

HAL is a multi-disciplinary open access archive for the deposit and dissemination of scientific research documents, whether they are published or not. The documents may come from teaching and research institutions in France or abroad, or from public or private research centers.
L'archive ouverte pluridisciplinaire HAL, est destinée au dépôt et à la diffusion de documents scientifiques de niveau recherche, publiés ou non, émanant des établissements d'enseignement et de recherche français ou étrangers, des laboratoires publics ou privés. 


\section{Author's Accepted Manuscript}

On the dynamics of the growth plate in primary ossification

A. Fasano, M.A. Herrero, J.M. López, E. Medina

PII:

S0022-5193(10)00273-0

DOI: doi:10.1016/j.jtbi.2010.05.030

Reference:

YJTBI 6009

To appear in: $\quad$ Journal of Theoretical Biology

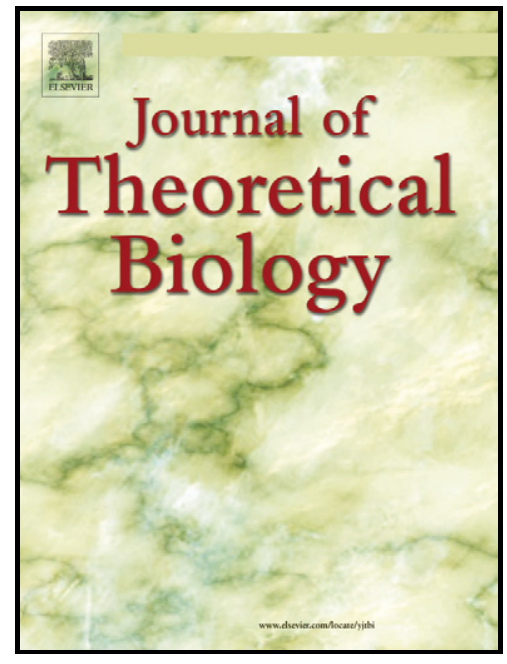

www.elsevier.com/locate/yjtbi

Received date: 15 January 2010

Revised date: $\quad 28$ April 2010

Accepted date: 25 May 2010

Cite this article as: A. Fasano, M.A. Herrero, J.M. López and E. Medina, On the dynamics of the growth plate in primary ossification, Journal of Theoretical Biology, doi:10.1016/j.jtbi.2010.05.030

This is a PDF file of an unedited manuscript that has been accepted for publication. As a service to our customers we are providing this early version of the manuscript. The manuscript will undergo copyediting, typesetting, and review of the resulting galley proof before it is published in its final citable form. Please note that during the production process errors may be discovered which could affect the content, and all legal disclaimers that apply to the journal pertain. 


\title{
On the dynamics of the growth plate in primary ossification
}

\author{
A. Fasano ${ }^{\text {a }}$, M.A. Herrero ${ }^{\text {b }}$,J.M. López ${ }^{\text {c }}$, E. Medina $^{\mathrm{d}}$ \\ ${ }^{a}$ Dipartimento di Matematica, Università di Firenze, Viale Morgagni 67/A, 50134 Firenze, \\ Italy. \\ ${ }^{b}$ IMI and Departamento de Matemática Aplicada, Facultad de Matemáticas, Universidad \\ Complutense, Plaza de las Ciencias s/n. 28040 Madrid, Spain. \\ ${ }^{c}$ Departamento de Morfología y Biología Celular, Facultad de Medicina, Universidad de \\ Oviedo, 33006 Oviedo, Spain. \\ ${ }^{d}$ Departamento de Matemáticas, Universidad de Cádiz. 11510 Puerto Real, Cádiz, Spain.
}

\begin{abstract}
In this work a mathematical model for the interaction of two key signalling molecules in rat tibia ossification is presented and discussed. The molecules under consideration are Indian hedgehog (Ihh) and parathyroid hormone-related peptide (PTHrP). These are known to be major agents in the dynamics of the so-called Growth Plate, where transition from pristine cartilage to advancing bone takes place. Our model consists in a steady-state linear approximation to a Reaction-Diffusion system where only diffusion and absorption mechanisms are retained. Estimates on some system parameters are given, on the basis of the knowledge of a few measurable quantities. This allows for explicitly solving our model, whereupon a discussion on robustness and regulatory properties thereof is provided. In particular, we show that the size of the Proliferative Zone in the Growth Plate is rather insensitive to variations in the flux coefficients for Ihh and PTHrP at their boundaries. Besides, we also show that the model is also insensitive to large changes in the (comparatively small) critical value of the PTHrP concentration which marks the transition form Proliferative to Hyperthropic Regions within the Growth Plate. These results hold irrespective of the particular diffusivities selected for Ihh and PTHrP.
\end{abstract}

\section{Introduction}

Ossification, the formation of bone tissue from a preexisting cartilage template, is a key stage in vertebrate development. The skeleton, which in humans contains over two hundred pieces of different sizes and shapes, performs a number of important functions. For instance, it provides a protecting frame for internal organs, and it transmits the force produced by muscle contraction in

Email addresses: fasano@math.unifi.it (A. Fasano), Miguel_Herrero@mat.ucm.es (M.A. Herrero), jmlopez@uniovi.es (J.M. López), elena.medina@uca.es (E. Medina) 
the course of movement. In this paper we investigate a key ossification process, whereby bone tissue is produced behind an advancing front and moves into a pre-existing cartilage template. More precisely, we aim at gaining insight into the manner in which cartilage cells (chondrocytes) determine the size of the Proliferative Zone (PZ), a cartilage region where cell proliferation takes place before chondrocytes undergo hypertrophy as a previous step to their replacement by invading bone tissue. The Proliferative Zone is located between two interfaces, separating it from the Resting Zone (RZ), where chondrocytes are generated, and from the Hyperthropic Zone (HZ), where proliferation is arrested, and the former cells enlarge in size before being overrun by advancing bone.

To describe this process, we shall make use of a comparatively simple mathematical model, to be explained and discussed below. The core of the model is a reaction-diffusion system describing how chemical signaling regulates the cell activity via an internal loop, which eventually determines the growth dynamics. Among the specific goals of the present study, we list: (i) to estimate some of the basic parameters of the process from the knowledge of measurable quantities, and (ii) to describe how the size of the Proliferative Zone depends upon variations on such parameters. As a preliminary step, we next provide a short summary of the basic features of ossification processes.

\subsection{Endochondral ossification: a process leading to long bone formation}

According to the manner in which it proceeds, mammalian ossification can be divided in two main types. A first one is intramembranous ossification, where bones grow by peripheral addition of new bone tissue at some osteogenic fronts. This is the way in which skull bones are made. A second type, endochondral ossification, is responsible for the formation of most vertebrate bones starting from a cartilage template. It involves two tightly coupled phenomena: continuous and vectorial production of cartilage tissue, and replacement of part of the cartilage by bone tissue through an invasive process. Endochondral ossification allows for a high rate of longitudinal growth, as required for the formation of long bones. In this last case, two coordinated stages can be distinguished, and are briefly recalled below.

Shortly after cartilage differentiation, chondrocytes in the central region of the template start growing larger in size, thus undergoing hypertrophy. Hypertrophic chondrocytes secrete, and organize, a particular type of extracellular matrix, ECM. Then periosteal buds containing blood vessels and mesenchymal cells invade the middle of the template. Such angiogenic process is instrumental in replacing cartilage by bone, and creates a primary ossification centre, that subsequently moves simultaneously into the distal areas of the cartilage template. This process is called primary ossification, and the whole zone including $\mathrm{RZ}, \mathrm{PZ}, \mathrm{HZ}$ is commonly termed as the Growth Plate (GP).

Once primary ossification sets in, cartilaginous areas at both distal sides develop an angiogenic process of their own. In this way, blood vessels penetrate distal peripheries to move inwards, thus producing secondary ossification centres at both ends of the template, which subsequently evolve much as primary centres do. This is known as secondary ossification, which happens to 
be strictly coordinated with primary ossification, to eventually result in almost total replacement of cartilage by bone, except for a thin region near the distal boundaries where periarticular cartilage remains. This last is crucial to allow for movable joints between adjacent bones. Both processes are represented in Figure 1 below. The reader is referred to [1], [2], [3], [4] and [5] for additional details.
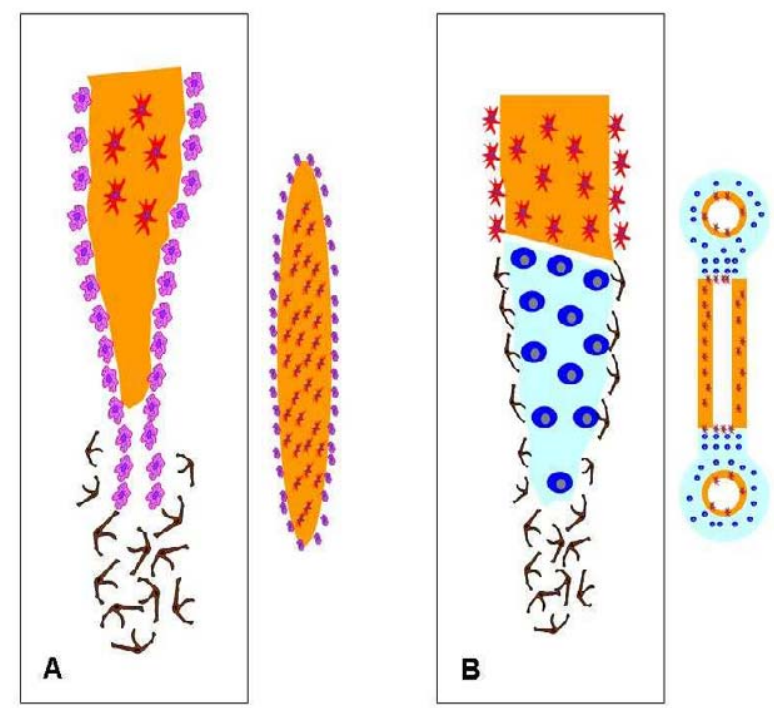

Figure 1: Diagram of stages of intramembranous (A) and endochondral (B) ossification. Mesenchymal cells (brown) are the precursor cells in both cases. In intramembranous ossification (A), mesenchymal cells directly differentiate into osteoblasts (purple) and these cells begin the secretion of mineralized matrix (orange). When a osteoblast is completely surrounded by matrix it differentiates to mature osteocytes (red). In a subsequent stage (inset A) most cells have differentiated into osteocytes and undifferentiated cells are solely located at the border of the bone. In endochondral ossification (B), mesenchymal cells differentiate into an intermediate cell type, the chondrocyte (blue) which form a temporary structure that is subsequently invaded by osteoblasts at both ends of the bone according to a well-defined spatial pattern (inset B).

Longitudinal bone growth thus depends on the activity of the growth plate that in particular leads to continuous production of new cell and cartilage matrix. While this phenomenon simultaneously proceeds in both opposite directions from the equatorial region, from now on we shall refer only to one of these two directions for definiteness. Chondrocytes of the growth plate are arranged into columnar stacks whose axes cluster about a vertical axis of anisotrophy. In a vertical column the chondrocyte life cycle is recapitulated, since chondrocytes 


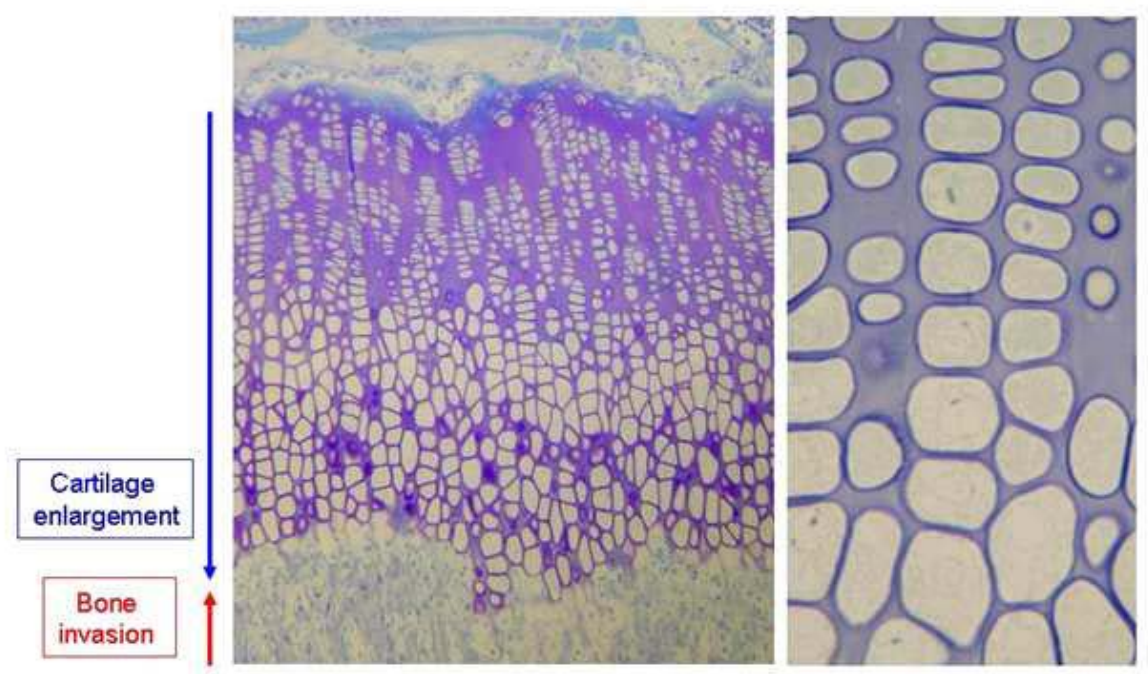

Figure 2: (Left) Section of the proximal tibial Growth Plate showing the different sizes of hypertrophic (bottom) and proliferative (top) chondrocytes. (Right) A magnification of one of the vertical columns which make up the Growth Plate, distinctly showing different types of chondrocytes along a vertical direction

display a continuous structural variation that reflects successive maturational stages of a cell-differentiation cycle (cf. Figure 2). Maturation occurs synchronously for cells located at the same horizontal level in different columns and this gives rise to distinct horizontal strata. The layer nearest the epiphysis (i.e., the more distal part, farther form the centre of the cartilage template) is narrow and irregularly contoured and is referred to as the Resting Zone. Chondrocytes in this zone are small in size, irregularly arranged and rarely divide. Further toward the metaphysis (i.e, the equatorial zone), chondrocytes undergo rapid division which increases the cell pool, thus contributing to the widening of the Growth Plate. Daughter chondrocytes from a cell division process lie one above the other, and this is of major importance for the formation of vertical columns and unidirectional growth of the bone. After having run through a certain number of cell divisions, chondrocytes lose their capacity to divide and begin to enlarge to become hypertrophic cells. Cell volume increase during hypertrophy results in particular in Growth Plate widening. Matrix synthesis during both proliferative and hypertrophic stages is a third component of cartilage expansion. In isolation, chondrocyte proliferation, cell-volume increase and matrix synthesis would lead to progressive enlarging of the Growth Plate. However, cartilage increase is balanced by a coordinated process of cartilage resorption and replacement by osseous tissue at a moving front, the epiphysealmetaphyseal interface. A strict coordination between the proceses of cartilage enlargement, cartilage resorption and osseous tissue formation is required for normal bone growth. 


\subsection{The dynamics of the Growth Plate: The Ihh/PTHrP regulatory loop}

Bone growth rate is dependent on the chondrogenic activity of the Growth Plate (GP). Different growth rates in different bones and/or in distinct developmental stages of the same bone are determined by differences in the rate of progression of chondrocytes through their differentiation cycle. Differentiation of Growth Plate chondrocytes involves a basic sequence of steps each of them having a wide range of quantitative variation. Parameters like the cell cycle time, the number of cellular divisions before the beginning of hypertrophy, the degree of organization into columns of cells, the time duration of hypertrophy, the rate of increase in cell volume during hypertrophy, the net matrix production and the rate of matrix mineralization could vary at different stages, and this determines different bone growth rates.

The extent of the different phases of the chondrocyte differentiation process as well as the replacement of cartilage by bone must be tightly regulated. Regulation must ensure not only the generation of the correct pattern of chondrocyte maturation but also the accurate quantitative values for each stage. In this way, it is well known that although regulation of Growth Plate is under a set of systemic, long-range control mechanisms, locally mediated control systems play a key role in GP evolution [6]. There are many different factors affecting the activity of chondrocytes, and it is the complex interplay of all these factors that determines their behaviour. The Indian hedgehog (Ihh)/ parathyroid hormone-related protein $(\mathrm{PTHrP})$ signaling pathway is a key regulator of these processes by forming a feedback loop to control the rate at which chondrocytes undergo maturation [3], [7], [8]. Indeed, it is well known that PTHrP is produced by periarticular chondrocytes and perichondral cells of the articular surface and acts directly on columnar proliferating chondrocytes to maintain the proliferative state and to block hypertrophic differentiation. Chondrocytes located close to the periarticular perichondrium (i.e, farther from the equatorial region) are exposed to high levels of PTHrP because of their relative proximity to the source, and such high concentrations inhibit chondrocyte hypertrophy. By contrast, chondrocytes farther from the articular surface are exposed to a lower concentration of PTHrP and thus undergo hypertrophic differentiation. PTHrP synthesis depends on, and is positively regulated by, Ihh which is expressed when proliferating chondrocytes differentiate into prehypertrophic chondrocytes. Thus, Ihh signals the relative position of prehypertrophic and hypertrophic chondrocytes to the periarticular Growth Plate. See references [7], [8] for further details on this regulatory loop, which is summarised in Figure 3 below.

As to the precedings of this work, a number of mathematical models have been proposed to describe chemical signalling among the various regions of the Growth Plate. Among them, we should mention [9] and [10] where ReactionDiffusion systems for the interaction among Ihh and PTHrP are postulated. While these articles have provided a basic inspiration for this work, our approach and that followed in [9] and [10] differ in that we have selected a simpler model, for which all parameters can be measured from experimental data with the help of the solutions of the mathematical model itself. Thus no further indirect 
estimates are required in order to fully discuss the behaviour of solutions, which yield the predicted values for the Ihh and PTHrP concentrations along the Proliferative Zone.

At this stage it should be noted that however important chemical signalling can be, it only constitutes one of the several mechanisms involved in the process of bone formation through endochondral ossification. For instance, a step further has been given in [11], where the coupling between cell density and chemical cues production has been considered. These authors formulated a model in which cell dynamics proceeds along a preferential, anisotropic direction (as it actually happens in the actual ossification process) which is in turn coupled to the Ihh and PTHrP concentrations, the latter being regulated by a suitable Reaction-Diffusion (RD) system. This work opens the way for further exploring the interaction among RD signalling models and the mechanics of bone growth (see also [12] for recent related work in a different context). However, the number of parameters appearing in the model is considerably larger than in our case, which leaves open the question of how these could be possibly determined from available experimental data. We wish also mention that RD models (without mechanical coupling) have also been used in [13] to describe secondary ossification, a process which, as we have remarked before, occurs at a different place than (but is finely tuned to) primary ossification, which is our main concern here.

\subsection{Aim of the paper}

The aim of the present work is to develop a mathematical model to describe the activity of the Ihh / PTHrP regulatory loop in the Proliferative Zone of the Growth Plate, and the comparative importance of the agents involved, as a function of a small number of key parameters.

More precisely, the plan of this paper is as follows. In Section 2 below, we describe and solve our mathematical model for the Ihh/PTHrP interaction in the Proliferative Zone (PZ). To this end some experimental results needed to identify parameters in the model are either recalled or provided.

We then discuss in Section 3 how the length of the Proliferative Zone is affected by small variations in the model coefficients, or by the Ihh/PTHrP concentrations at the $\mathrm{RZ} / \mathrm{PZ}$ and $\mathrm{PZ} / \mathrm{HZ}$ interfaces. In particular, we show that variations in the flow parameters in the model are largely damped out by the Ihh/PTHrP feedback loop, whereas fluctuations of the concentrations of such molecules at the RZ/PZ interface have opposite effects on the length of the $\mathrm{PZ}$ region. Moreover, the impact of variations in a particularly small parameter ( $c_{P H}$, the concentration of PTHrP at the PZ/HZ interface) is assessed. Our results are presented for three different age groups (21,35 and 80 days old rats). Such choice is motivated by the well-known fact that the Growth Plate dynamics is strongly age-dependent, which is in turn related to the influence of additional signalling molecules (e.g. sexual hormones) which are not dealt with 


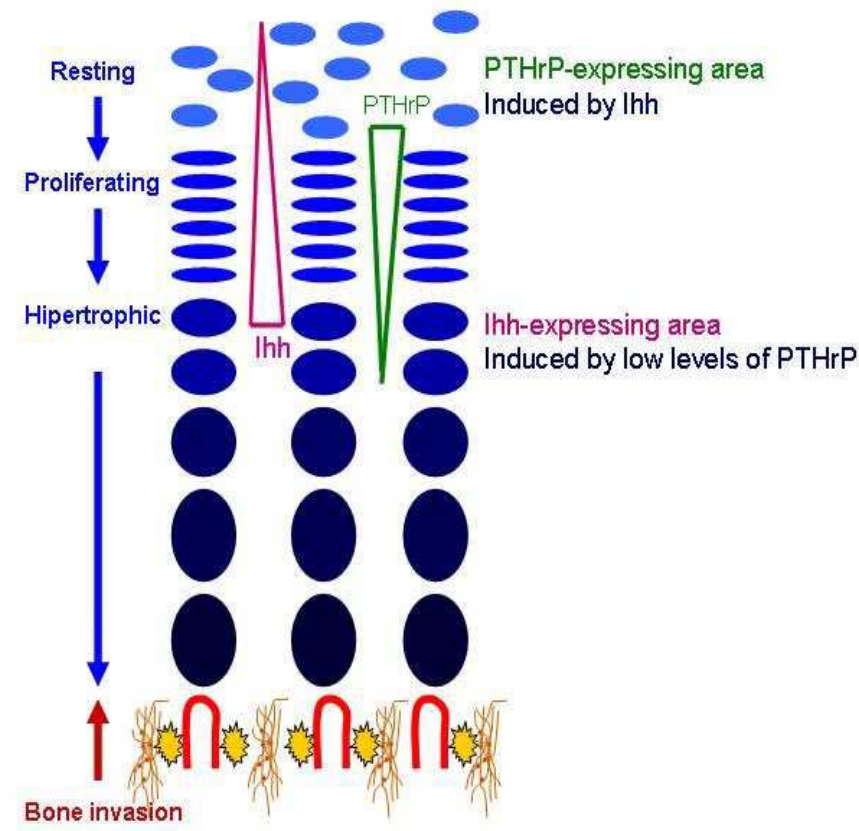

Figure 3: Thh is expressed in early hypertrophic chondrocytes and induces expression of PTHrP by resting chondrocytes. PTHrP keeps chondrocytes in a proliferating state, inhibiting expression of Ihh. Chondrocytes undergo hypertrophic differentiation and begin to express Ihh only when PTHrP drops below a threshold level. Thus Ihh and PTHrP form a negative feedback loop since increased production of Ihh results in its subsequent reduction by the system. This mechanism self-regulates the distance between the boundaries of the Resting Zone RZ (PTHrP-expressing area) and the Hyperthrophic Zone HZ (Ihh-expressing area). In our approach, the RZ/PZ and the $\mathrm{PZ} / \mathrm{HZ}$ transition regions, which are assumed to be thin enough, are considered as signalling interfaces, a terminology to be kept in the sequel.

here. A number of conclusions are then listed in our final Section 4. On the other hand, as we shall presently see, our model relies only on absorption and diffusion as mass transport mechanisms, thus discarding other such processes as convection. A discussion on the negligible character of the latter in our current setting is postponed to Appendix A at the end of the paper.

\section{The dynamics of the Growth Plate}

We next state, and solve, the mathematical model that we propose to describe the Ihh/PTHrP signalling loop at the Growth Plate.

A remarkable fact concerning primary ossification is that many key aspects of such process can be considered in a one-dimensional setting, since chondrocytes are arranged along vertical columns, and lines at same distance from the advancing bone front correspond to a similar cell maturation state (cf. for instance [3]). 
In what follows we shall keep track of the Growth Plate dynamics along a single column of chondrocytes, but all quantities entering the model should be considered as averages over the whole set of columns. We focus our attention on the GP region, and more precisely in the Proliferative Zone (PZ) therein. For modelling purposes, it is convenient to select a reference frame in which the boundary between the $\mathrm{PZ} / \mathrm{HZ}$ boundary remains at rest. More precisely, we take a longitudinal coordinate $\hat{z}$ such that $\hat{z}=0$ is located at the position of such an interface. On the other hand, the RZ-PZ transition is assumed to occur at $\hat{z}=\Gamma_{P}$, with $\mathrm{HZ}$ ending at $\hat{z}=-\Gamma_{H}$. In this reference frame, chondrocytes move from right to left. This situation is depicted in Figure 4 below:

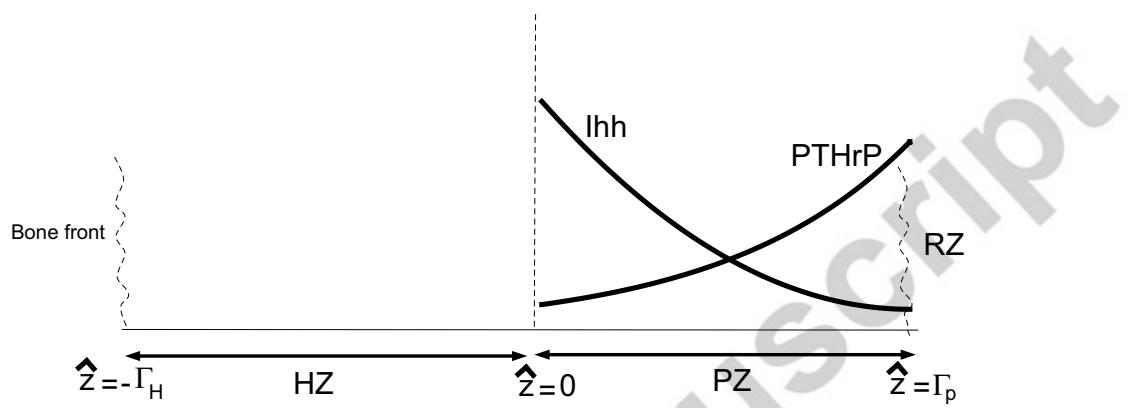

Figure 4: From right to left, Resting Zone (RZ) and Proliferative Zone (PZ) are shown. Note that decreasing values of $\hat{z}$ lead to transition from the Proliferative to the Hypertrophic Zone. Ihh/PTHrP gradients are represented in the PZ Region

\subsection{A mathematical model for Ihh/PTHrP interaction at the Proliferative Re- gion}

To describe in a precise manner the nature of the interaction between Ihh and PTHrP signalling in the Growth Plate, some quantitative models have been considered (cf. for instance [9], [10], [11], [12], [13]). As in those articles, transport of PTHrP and Ihh will be assumed to occur basically by diffusion through the liquid permeating the ECM, and by absorption. The latter process has two basic components. The first one takes place over chondrocytes, and is regulated by the kinetics of binding to specific receptors at the cell membrane. On the other hand, absorption takes also place at the ECM, the corresponding rate being in principle different from that for receptor binding. These two types of mechanisms will be represented by means of a single, effective absorption term in the model that follows. On its turn, convection will be assumed to be negligible. Justification for this last hypothesis is postponed to Appendix A.

We next state our mass balance equations in the proliferative zone. To this end, we respectively denote by $\hat{c}_{p}, \hat{c}_{i}$ the concentrations of PTHrP and Ihh and write:

$$
-D \frac{d^{2} \hat{c}_{i}}{d \hat{z}^{2}}+\delta_{i} \hat{c}_{i}=0, \quad 0<\hat{z}<\Gamma_{P},
$$




$$
-D \frac{d^{2} \hat{c}_{p}}{d \hat{z}^{2}}+\delta_{p} \hat{c}_{p}=0, \quad 0<\hat{z}<\Gamma_{P}
$$

together with the boundary conditions:

$$
\begin{gathered}
-\left.D \frac{d \hat{c}_{i}}{d \hat{z}}\right|_{\hat{z}=0}=\hat{\gamma}_{i}, \\
\left.\frac{d \hat{c}_{i}}{d \hat{z}}\right|_{\hat{z}=\Gamma_{P}}=0, \\
\left.D \frac{d \hat{c}_{p}}{d \hat{z}}\right|_{\hat{z}=\Gamma_{P}}=\hat{\alpha}_{p} \hat{c}_{i}\left(\Gamma_{P}\right) .
\end{gathered}
$$

At the transition between the $\mathrm{PZ}$ and $\mathrm{HZ}$ regions we assume that

$$
\hat{c}_{p}(0)=c_{P H}
$$

where $c_{P H}$, the concentration of $\mathrm{PTHrP}$ at the $\mathrm{PZ} / \mathrm{HZ}$ interface is considered as a model parameter, and:

$$
\left.\frac{d \hat{c}_{p}}{d \hat{z}}\right|_{\hat{z}=0}=0
$$

Before solving the problem consisting in (2.1)-(2.7) some remarks on the motivation thereof are in order. To begin with, (2.1), (2.2) represent a steady-state approximation to a diffusion-absorption process. As in previous related work (cf. for instance [14]) the diffusion coefficients for Ihh and PTHrP have been assumed equal for simplicity. This hypothesis is motivated by the fact that both substances possess similar molecular weights (cf. [15], [16], [17]), although no direct estimates on their diffusivities along the Growth Plate seem to be available; for instance, the value for the common diffusion coefficient selected in [14] (and recalled in (2.26) below) has been determined for bovine albumin, a substance of similar molecular weight to that of Ihh and PTHrP. There are, however, authors who have selected different diffusivities for both substances in their studies (see for instance [11], [13]). In those works, the authors deal with models including a large number of parameters, whose values cannot be determined in a unique way, and propose diffusivity choices which, being in some cases arbitrary, are compatible with standard activation-inhibition assumptions as explained for instance in [18]. An important fact concerning our approach is that, as we shall discuss in detail later on, our basic results concerning regulatory properties of the Proliferating Zone are independent of the particular values chosen for these coefficients, and in particular selecting both diffusivities equal or different does not change such results. Condition (2.3) corresponds to the assumption that a flux of Thh is produced at the moment chondrocytes leave PZ to enter HZ, that is, at the PZ/HZ interface (see [19], [20], [8]). Since flux is thought to occur at such precise threshold, it is natural to assume it to be constant, as we shall do in the sequel. On its turn, (2.4) states that Ihh diffusion beyond $\hat{z}=\Gamma_{P}$ is assumed to be negligible (the absence of flux can be justified by the occurrence 
of secondary ossification, a process somehow symmetric taking place within the epiphysis).

Condition (2.5) states that, at the RZ/PZ boundary, a flux of PTHrP is produced, which is proportional to the concentration of Ihh there. This hypothesis corresponds to current assumptions on the way in which PTHrP production at the interface of the Resting Zone is stimulated by Ihh concentration there (see for instance [8], [19], [20], [21]) .

Finally, by (2.7) we mean that diffusion of PTHrP is basically symmetric in $\mathrm{PZ}$ and in $\mathrm{HZ}$.

\subsection{Solving the PZ model}

To proceed further, we introduce non-dimensional variables as follows:

$$
z=\frac{\hat{z}}{\Gamma_{P}}, \quad c_{i}=\frac{\hat{c}_{i}}{c_{P H}}, \quad c_{p}=\frac{\hat{c}_{p}}{c_{P H}}, \quad \gamma_{i}=\hat{\gamma}_{i} \frac{\Gamma_{P}}{D c_{P H}}, \quad \alpha_{p}=\hat{\alpha}_{p} \frac{\Gamma_{P}}{D}
$$

A quick check reveals that, in the new variables (2.8), (2.1)-(2.7) transforms into

$$
\frac{d^{2} c_{i}}{d z^{2}}=\Omega_{i}^{2} c_{i} \quad \text { for } 0<z<1 \quad \text { where } \quad \Omega_{i}^{2}=\frac{\Gamma_{P}^{2} \delta_{i}}{D}=\tau_{D} \delta_{i}
$$

with $\tau_{D}$ being a characteristic diffusive time given by $\tau_{D}=\frac{\Gamma_{P}^{2}}{D}$,

$$
\begin{gathered}
\frac{d^{2} c_{p}}{d z^{2}}=\Omega_{p}^{2} c_{p} \quad \text { for } 0<z<1 \quad \text { where } \Omega_{p}^{2}=\frac{\Gamma_{P}^{2} \delta_{p}}{D}=\tau_{D} \delta_{p}, \\
\left.\frac{d c_{i}}{d z}\right|_{z=0}=-\gamma_{i}, \\
\left.\frac{d c_{i}}{d z}\right|_{z=1}=0, \\
\frac{d c_{p}}{d z}(1)=\alpha_{p} c_{i}(1), \\
c_{p}(0)=1, \\
\frac{d c_{p}}{d z}(0)=0 .
\end{gathered}
$$

Equations (2.9)-(2.15) can be explicitly solved. Indeed, on trying $c_{i}(z)=$ $a_{1} \sinh \left(\Omega_{i} z\right)+a_{2} \cosh \left(\Omega_{i} z\right)$ and $c_{p}(z)=b_{1} \sinh \left(\Omega_{p} z\right)+b_{2} \cosh \left(\Omega_{p} z\right)$ where $\sinh (s)=\frac{e^{s}-e^{-s}}{2}, \cosh (s)=\frac{e^{s}+e^{-s}}{2}$, one obtains after some elementary calculations that:

$$
c_{i}(z)=\frac{\gamma_{i}}{\Omega_{i}}\left(\operatorname{coth} \Omega_{i} \cosh \left(\Omega_{i} z\right)-\sinh \left(\Omega_{i} z\right)\right),
$$




$$
c_{p}(z)=\cosh \left(\Omega_{p} z\right)
$$

where $\operatorname{coth} \Omega_{i}=\frac{\cosh \Omega_{i}}{\sinh \Omega_{i}}$. A link between $c_{i}(z)$ and $c_{p}(z)$ is given by $(2.13)$ which yields

$$
\Omega_{i} \Omega_{p} \sinh \Omega_{i} \sinh \Omega_{p}=\alpha_{p} \gamma_{i} .
$$

Equation (2.18) provides a compatibility condition for the length of the proliferative zone $\Gamma_{P}$ in terms of the parameters of the original problem (2.1)-(2.7). Indeed, on going back to dimensional variables (cf. (2.8)), one readily sees that (2.18) transforms into

$\omega_{i} \omega_{p} \sinh \left(\omega_{i} \Gamma_{P}\right) \sinh \left(\omega_{p} \Gamma_{P}\right)=\frac{\hat{\alpha}_{p} \hat{\gamma}_{i}}{D^{2} c_{P H}}, \quad$ where $\quad \omega_{p}=\left(\frac{\delta_{p}}{D}\right)^{\frac{1}{2}}, \omega_{i}=\left(\frac{\delta_{i}}{D}\right)^{\frac{1}{2}}$

\subsection{Estimating parameters from experimental data}

We next set out to estimate the coefficients in (2.16)-(2.18), as well as in the corresponding dimensional formulae. To this end we shall begin by making use of some available experimental results. More precisely the following values have been reported in [10]; see also [22], [23] and [7].

$$
\begin{aligned}
& \hat{c}_{i}(0)=0.5 \mu M ; \quad \hat{c}_{i}\left(\Gamma_{P}\right)=0.2 \mu M ; \quad \hat{c}_{p}\left(\Gamma_{P}\right)=0.3 \mu M ; \\
& \hat{c}_{p}(0)=c_{P H}=0.1 n M .
\end{aligned}
$$

Note that the value assigned to $c_{P H}$ is significantly smaller than the remaining three figures. Recalling (2.8), one readily deduces from (2.20) that

$$
c_{i}(0)=5 \cdot 10^{3} ; \quad c_{i}(1)=2 \cdot 10^{3} ; \quad c_{p}(1)=3 \cdot 10^{3} ; \quad c_{p}(0)=1 .
$$

Plugging (2.21) into (2.16) yields:

$$
\frac{\gamma_{i}}{\Omega_{i}} \operatorname{coth} \Omega_{i}=5 \cdot 10^{3}, \quad \frac{\gamma_{i}}{\Omega_{i} \sinh \Omega_{i}}=2 \cdot 10^{3},
$$

whence

$$
\cosh \Omega_{i}=2.5
$$

which in turns yields the estimate

$$
\Omega_{i}=1.57 \text {. }
$$

Moreover from (2.22) and (2.23) we obtain:

$$
\gamma_{i}=7.18 \cdot 10^{3}
$$

We are now in a position to estimate our first dimensional parameter $\hat{\gamma}_{i}$ in $(2.3)$. Since by (2.8)

$$
\hat{\gamma}_{i}=\frac{D c_{P H}}{\Gamma_{P}} \gamma_{i},
$$


and we have already recalled an estimate for $c_{P H}$ (cf. (2.20)), we shift our attention to $\Gamma_{P}$, we shall make use of the following values

$$
\begin{aligned}
& \Gamma_{P}=180 \mu \mathrm{m} \quad \text { for the age of } 21 \text { days, } \\
& \Gamma_{P}=160.6 \mu \mathrm{m} \text { for the age of } 35 \text { days, } \\
& \Gamma_{P}=137.5 \mu \mathrm{m} \text { for the age of } 80 \text { days. }
\end{aligned}
$$

This figures are obtained from experimental results for rat tibia in three age groups, which are reported below in Table 1

TABLE 1
\begin{tabular}{|l|cc|cc|cc|}
\hline Parameter & $\mathbf{2 1}$ days of age & \multicolumn{3}{c}{$\mathbf{3 5}$ days of age } & $\mathbf{8 0}$ days of age \\
\hline Growth plate height $(\mu \mathrm{m})$ & 736,3 & 61,4 & 589,5 & 45,2 & 297,7 & 25,1 \\
Height $(\mu \mathrm{m})$ of the Resting & 47,5 & 7,8 & 38,4 & 5,1 & 25,6 & 7,2 \\
Height $(\mu \mathrm{m})$ of Proliferating & 179,8 & 12,1 & 160,6 & 9,2 & 137,5 & 14,9 \\
Height $(\mu \mathrm{m})$ of Upper Hypertrophic & 254,5 & 27,9 & 195,2 & 19,8 & 67,3 & 15,9 \\
Height $(\mu \mathrm{m})$ of Lower Hypertrophic & 254,5 & 27,9 & 195,2 & 19,8 & 67,3 & 15,9 \\
\hline
\end{tabular}

Here SD stands for the standard deviation. To derive Table 1, three age groups of male Sprague-Dawley rats were used 21, 35 and 80 days after birth. Tissue collection and processing were performed as described in [25]. Analysis of longitudinal bone growth rates and proliferative activity were achieved. Proliferating chondrocytes were immunocytochemically identified by the 5-bromodeoxyuridine (BrdU) method. Proliferative activity was quantified by the percentage of BrdU-labeled cells within the proliferative stratum. Histomorphological methods were carried out as previously reported. Basically, sections were stained with toluidine blue and photographed in the light microscope first at a low magnification (X230). On these prints, growth plates were divided into stem cell, proliferating and hypertrophic zones according to morphological criteria. The hypertrophic zone was subdivided into an upper (H1) and lower (H2) half of equal size. The heights the growth plate and its different zones were estimated by point counting.

As to the diffusion coefficient $D$, we select for definiteness

$$
D=50 \mu \mathrm{m}^{2} / \mathrm{s} .
$$

The choice of this figure is motivated by the value reported in the experimental study conducted in [14]. It should be noted, however, that in such work the diffusivity of bovine albumin in the Growth Plate was considered. Based on the similarity of molecular weights, it was assumed that Ihh and PTHrP diffusivities should be similar to that of such substance. We are not aware, though, of any direct study on Ihh/PTHrP diffusion properties along the Growth Plate. As we have already remarked at the end of paragraph 2.1 , we shall presently show that our key results are not affected by the particular choice made in (2.26).

Summing all the results up we obtain the estimates:

$\hat{\gamma}_{i}=0.199 \mu M \cdot \mu m \cdot s^{-1}, \quad 0.224 \mu M \cdot \mu m \cdot s^{-1}, \quad$ and $\quad 0.261 \mu M \cdot \mu m \cdot s^{-1}$, 
for the ages of 21,35 and 80 days respectively. In a similar manner, we deduce from $(2.17)$ that $\cosh \Omega_{p}=3 \cdot 10^{3}$, whence we take

$$
\Omega_{p}=8.70
$$

We now estimate $\alpha_{p}$ from (2.18) to obtain

$$
\alpha_{p}=13.0
$$

whence $\hat{\alpha}_{p}=\alpha_{p} \frac{D}{\Gamma_{P}}$ can be taken to be

$$
\hat{\alpha}_{p}=3.62 \mu \mathrm{m} \cdot \mathrm{s}^{-1}, 4.06 \mu \mathrm{m} \cdot \mathrm{s}^{-1}, \text { and } 4.75 \mu \mathrm{m} \cdot \mathrm{s}^{-1} \text {, }
$$

for the ages of 21, 35 and 80 days respectively. Finally $\delta_{i}$ and $\delta_{p}$ are determined through the relations (2.9)-(2.10) as

$$
\delta_{p}=\frac{D \Omega_{p}^{2}}{\Gamma_{P}^{2}}, \quad \delta_{i}=\frac{D \Omega_{i}^{2}}{\Gamma_{P}^{2}}
$$

whence

$$
\begin{aligned}
& \delta_{i}=3.79 \cdot 10^{-3} s^{-1}, \quad 4.76 \cdot 10^{-3} s^{-1} \text { and } 6.49 \cdot 10^{-3} s^{-1} \text {, } \\
& \delta_{p}=11.7 \cdot 10^{-2} s^{-1}, \quad 14.7 \cdot 10^{-2} s^{-1} \text { and } 2.00 \cdot 10^{-1} s^{-1} \text {. }
\end{aligned}
$$

for the ages of 21, 35 and 80 days respectively.

\section{The size of the $\mathrm{PZ}$ region: regulatory properties}

In this Section we begin by discussing how variations on parameters appearing on our Ihh/PTHrP model reflect on the length of PZ. To this end, we shall use a perturbative approach, and consider in each case small changes in the coefficients involved.

\subsection{Dependence of $\Gamma_{P}$ on small perturbations of the flux coefficients}

We first show that the size of PZ is robust (that is, comparatively insensitive) with respect to small variations in the flux coefficients in (2.1)-(2.7). To this end, let us denote by $\Gamma_{P}^{0}$ the values of $\Gamma_{P}$ corresponding to the values of $\hat{\alpha}_{p}$, $\hat{\gamma}_{i}$ specified in our former Section. In particular for the ages of 21, 35 and 80 days we have seen that $\Gamma_{P}^{0}=180,160.6$ and $137.5 \mu \mathrm{m}$ respectively. Suppose now that $\hat{\alpha}_{p}, \hat{\gamma}_{i}$ undergo small perturbations, so that their values are changed to $\hat{\alpha}_{p}\left(1+\epsilon_{p}\right), \hat{\gamma}_{i}\left(1+\eta_{i}\right)$ with $0<\epsilon_{p}, \eta_{i} \ll 1$. Let us denote by $\Gamma_{P}^{0}$ the original length of PZ and by $\Gamma_{P}^{0}(1+\xi)$ that of the resulting $\mathrm{PZ}$ corresponding to the new flux coefficient values. In the perturbed case, (2.19) reads:

$$
\omega_{p} \omega_{i} \sinh \left(\omega_{p} \Gamma_{P}^{0}(1+\xi)\right) \sinh \left(\omega_{i} \Gamma_{P}^{0}(1+\xi)\right)=\frac{\hat{\alpha}_{p}\left(1+\epsilon_{p}\right) \hat{\gamma}_{i}\left(1+\eta_{i}\right)}{D^{2} c_{P H}} .
$$


Expanding both sides by Taylor's formula, we obtain

$$
\begin{gathered}
\omega_{i} \omega_{p}\left(\sinh \left(\omega_{p} \Gamma_{P}^{0}\right) \sinh \left(\omega_{i} \Gamma_{P}^{0}\right)+\xi \omega_{p} \Gamma_{P}^{0} \cosh \left(\omega_{p} \Gamma_{P}^{0}\right) \sinh \left(\omega_{i} \Gamma_{P}^{0}\right)+\right. \\
\left.\xi \omega_{i} \Gamma_{P}^{0} \cosh \left(\omega_{i} \Gamma_{P}^{0}\right) \sinh \left(\omega_{p} \Gamma_{P}^{0}\right)+\mathcal{O}\left(\xi^{2}\right)\right)=\frac{\hat{\alpha}_{p} \hat{\gamma}_{i}}{D^{2} c_{P H}}\left(1+\epsilon_{p}+\eta_{i}+\epsilon_{p} \eta_{i}\right) .
\end{gathered}
$$

Thus, retaining only linear terms in (3.1), and substracting (2.19) from the resulting expression (with $\Gamma_{P}$ replaced by $\Gamma_{P}^{(0)}$ ), we eventually obtain that, to the lowest order:

$$
\xi \Gamma_{P}^{0}\left(\omega_{p} \operatorname{coth}\left(\omega_{p} \Gamma_{P}^{0}\right)+\omega_{i} \operatorname{coth}\left(\omega_{i} \Gamma_{P}^{0}\right)\right)=\epsilon_{p}+\eta_{i} .
$$

Recalling that

$$
\Gamma_{P}^{0} \omega_{p} \operatorname{coth}\left(\omega_{p} \Gamma_{P}^{0}\right)+\Gamma_{P}^{0} \omega_{i} \operatorname{coth}\left(\omega_{i} \Gamma_{P}^{0}\right)=\Omega_{p} \operatorname{coth} \Omega_{p}+\Omega_{i} \operatorname{coth} \Omega_{i},
$$

(cf. (2.19)), and taking advantage of the estimates obtained for $\Omega_{p}$ and $\Omega_{i}$, in our current case (cf. (2.23), (2.28)), we deduce from (3.2) that

$$
\xi=\frac{1}{10.4}\left(\epsilon_{p}+\eta_{i}\right)
$$

and therefore the relative perturbation in the length $\Gamma_{P}^{0}$ is one order of magnitude inferior to the relative perturbations in the flux coefficients $\hat{\alpha}_{p}, \hat{\gamma}_{i}$.

We next remark that the robustness result described in (3.4) is independent of the particular choice of equal diffusivities made in (2.26). Suppose for instance that we now consider that effective diffusivities of Ihh and PTHrP are respectively given by $D_{i}$ and $D_{p}$. Then, we have to replace $D$ by $D_{i}$ in $(2.1)$ and (2.3), and by $D_{p}$ in (2.2) and (2.5). If we next use the non-dimensional variables $z, c_{i}$ and $c_{p}$ given by the three first equations in (2.8), we obtain the system (2.9)-(2.15) where

$$
\Omega_{i}^{2}=\frac{\Gamma_{P}^{2} \delta_{i}}{D_{i}}, \quad \Omega_{p}^{2}=\frac{\Gamma_{P}^{2} \delta_{p}}{D_{p}}, \quad \gamma_{i}=\hat{\gamma}_{i} \frac{\Gamma_{P}}{D_{i} c_{P H}}, \quad \alpha_{p}=\hat{\alpha}_{p} \frac{\Gamma_{P}}{D_{p}} .
$$

Now, taking into account (2.16) and (2.17), it is clear that the values of the nondimensional parameters $\Omega_{i}, \Omega_{p}$ and $\gamma_{i}$ remain as in (2.23), (2.28) and (2.24). Thus, the different choice of diffusivities only modify the dimensional parameters $\delta_{i}, \delta_{p}, \hat{\gamma}_{i}, \hat{\alpha}_{p}$. On the other hand, from (3.2) and (3.3) we have that

$$
\xi=\frac{\epsilon_{p}+\eta_{i}}{\Omega_{p} \operatorname{coth} \Omega_{p}+\Omega_{i} \operatorname{coth} \Omega_{i}},
$$

which coincides with (3.4) independently of the values of the diffusivities. 
3.2. Dependence of $\Gamma_{P}$ on small perturbations of the boundary concentrations of PTHrP and Ihh

Suppose now that the concentrations of PTHrP and Ihh undergo small variations at the boundary of the proliferative zone. As before, let us denote by $\Gamma_{P}^{0}$ the original length of $\mathrm{PZ}$ and by $\Gamma_{P}^{0}(1+\xi)$ that of the resulting $\mathrm{PZ}$ corresponding to the new concentration values. Let the new non-dimensional concentrations be given by

$$
\bar{c}_{i}(0)=c_{i}(0)\left(1+\delta_{0}\right), \quad \bar{c}_{i}(1)=c_{i}(1)\left(1+\delta_{1}\right), \quad \bar{c}_{p}(1)=c_{p}(1)(1+\eta),
$$

where we are denoting with the superscript ${ }^{-}$those variables which correspond to the perturbed situation. Note also that the one-dimensional length $\bar{z}$ in (3.6) has been obtained by rescaling with respect to the new PZ size. Here we leave $c_{P H}$ unperturbed, postponing the analysis of the corresponding perturbation to the following subsection. On writing equations (2.16) and (2.17)

$$
\cosh \Omega_{i}=\frac{c_{i}(0)}{c_{i}(1)}, \quad \cosh \Omega_{p}=c_{p}(1)
$$

both in the perturbed and unperturbed case, one readily obtains

$$
\begin{aligned}
& \cosh \bar{\Omega}_{i}=\cosh \Omega_{i} \frac{1+\delta_{0}}{1+\delta_{1}}=\cosh \Omega_{i}\left(1+\delta_{0}-\delta_{1}\right)+\text { lower order terms, } \\
& \cosh \bar{\Omega}_{p}=\cosh \Omega_{p}(1+\eta)
\end{aligned}
$$

Besides, from (2.8) and (2.16) in $\hat{z}=0$ and $\hat{z}=\Gamma_{P}$, we obtain that

$$
\hat{\gamma}_{i}=\frac{D}{\Gamma_{P}^{(0)}} \Omega_{i} \sinh \Omega_{i} \hat{c}_{i}\left(\Gamma_{P}^{(0)}\right)
$$

and taking into account this equation in the perturbed and unperturbed cases we also see that

$$
\frac{\hat{\bar{\gamma}}_{i}}{\hat{\gamma}_{i}}=\frac{\bar{\Omega}_{i} \sinh \bar{\Omega}_{i}}{\Omega_{i} \sinh \Omega_{i}} \frac{1+\delta_{1}}{1+\xi} .
$$

On the other hand, it follows from (2.19) that

$$
\frac{\bar{\Omega}_{p} \bar{\Omega}_{i}}{\left(\Gamma_{P}^{0}\right)^{2}(1+\xi)^{2}} \sinh \bar{\Omega}_{p} \sinh \bar{\Omega}_{i}=\frac{\hat{\alpha_{p}} \hat{\bar{\gamma}}_{i}}{D^{2} c_{P H}},
$$

that using (3.9) can be rewritten as

$$
\bar{\Omega}_{p} \Omega_{i} \sinh \bar{\Omega}_{p} \sinh \Omega_{i}=\frac{\hat{\alpha}_{p} \hat{\gamma}_{i}}{D^{2} c_{P H}}\left(\Gamma_{P}^{0}\right)^{2}(1+\xi)\left(1+\delta_{1}\right),
$$

that comparing with (2.19) leads us to

$$
\frac{\bar{\Omega}_{p} \sinh \bar{\Omega}_{p}}{\Omega_{p} \sinh \Omega_{p}}=\left(1+\delta_{1}\right)(1+\xi) .
$$


Since, by (3.7) and (2.28) we have that, to the leading order

$$
\bar{\Omega}_{p}=\Omega_{p}+\eta\left(\operatorname{coth} \Omega_{p}\right)=\Omega_{p}\left(1+\frac{\eta}{\Omega_{p}}\right),
$$

and

$$
\sinh \bar{\Omega}_{p}=\sinh \left(\Omega_{p}+\eta\right)=\sinh \Omega_{p}\left[1+\left(\operatorname{coth} \Omega_{p}\right) \eta\right]=\sinh \Omega_{p}(1+\eta),
$$

(where we used that for values of $\Omega_{p}$ close to $\Omega_{p}=8.70$ we approximately have that $\operatorname{coth} \Omega_{p}=1$ ), equation $(3.12)$ reduces to

$$
\left(1+\frac{\eta}{\Omega_{p}}\right)(1+\eta)=\left(1+\delta_{1}\right)(1+\xi) .
$$

Retaining only leading terms we arrive at

$$
\xi=\left[\left(\frac{1}{\Omega_{p}}+1\right) \eta-\delta_{1}\right] .
$$

Then, in this range of approximations, the perturbed $\Gamma_{P}$ length is sensitive to variations on $c_{i}(1)$ and $c_{p}(1)$, but not to variations of the Ihh concentration at the $\mathrm{PZ} / \mathrm{HZ}$ interface. Again, this result is independent of the fact that the diffusivities are taken equal or different, and is also independent of their particular values. Indeed, by recalling (3.5), it is clear that if we take diffusivities $D_{i}$ and $D_{p}$ for Ihh and PTHrP respectively, we need to replace $D$ by $D_{i}$ into (3.8) and $D^{2}$ by $D_{i} D_{p}$ into (3.10) and (3.11). However, equations (3.9), (3.12) and (3.13) remain invariant.

\subsection{Negative regulation: sensitivity with respect to $c_{P H}$}

We have already remarked that parameter $c_{P H}$ stands out among other parameters in the model since it is comparatively quite small. Hence, any estimate on its precise value has to be taken with caution. Then, the question naturally arises of ascertaining whether changes in its value have a significant impact on the behavior of the solutions of the model. We shall consider below a particular case of the corresponding sensitivity analysis. More precisely,we next study the way in which changes in $c_{P H}$ translate into changes for $\delta_{p}$ and $\hat{\alpha}_{p}$ to keep the length of PZ invariant. To do that we consider values for $c_{P H}$ within the range $c_{P H} \in\left(10^{-5} \mu M, 10^{-3} \mu M\right)$ and obtain from our solution the required values for $\delta_{p}$ and $\hat{\alpha}_{p}$. The results are given in Figures 5 and 6 and in tables 2, 3 and 4 where $\delta_{p}$ is given in $s^{-1}$ and $\hat{\alpha}_{p}$ in $\mu \mathrm{m} / \mathrm{s}$. 


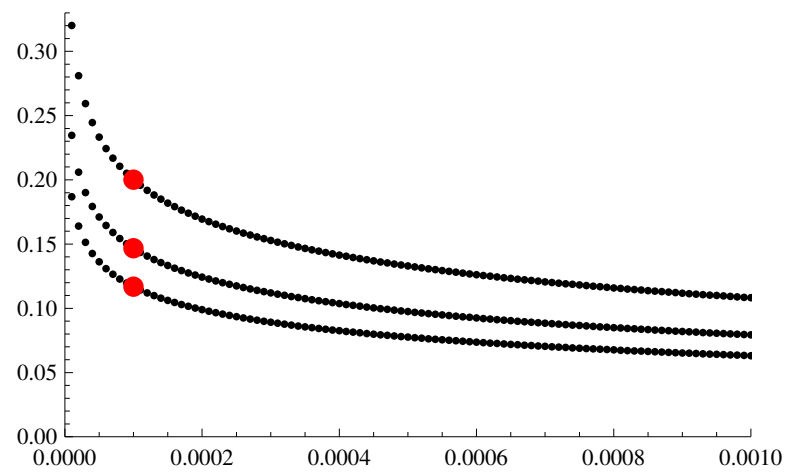

Figure 5: $\delta_{p}$ as a function of $c_{p H}$ for 21 (bottom), 35 (middle) and 80 (top) days of age respectively. Dots correspond to the value selected in (2.20)

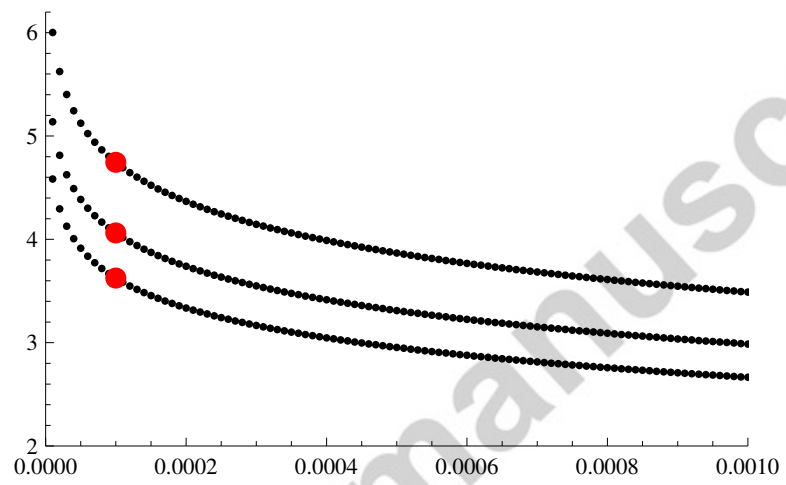

Figure 6: $\hat{\alpha}_{p}$ as a function of $c_{p H}$ for 21 (bottom), 35 (middle) and 80 (top) days of age respectively. Dots correspond to the value selected in (2.20)

Table 2: 21 days of age

\begin{tabular}{|c|ccccccccc|}
\hline$c_{P H}$ & $10^{-5}$ & $4 \cdot 10^{-5}$ & $7 \cdot 10^{-5}$ & $10^{-4}$ & $2 \cdot 10^{-4}$ & $3 \cdot 10^{-4}$ & $5 \cdot 10^{-4}$ & $7.5 \cdot 10^{-4}$ & $10^{-3}$ \\
\hline$\delta_{p}$ & 0.187 & 0.143 & 0.127 & 0.117 & 0.0989 & 0.0892 & 0.0776 & 0.0690 & 0.0631 \\
\hline$\hat{\alpha}_{p}$ & 4.58 & 4.01 & 3.77 & 3.62 & 3.34 & 3.17 & 2.95 & 2.79 & 2.67 \\
\hline
\end{tabular}

Table 3: 35 days of age

\begin{tabular}{|c|ccccccccc|}
\hline$c_{P H}$ & $10^{-5}$ & $4 \cdot 10^{-5}$ & $7 \cdot 10^{-5}$ & $10^{-4}$ & $2 \cdot 10^{-4}$ & $3 \cdot 10^{-4}$ & $5 \cdot 10^{-4}$ & $7.5 \cdot 10^{-4}$ & $10^{-3}$ \\
\hline$\delta_{p}$ & 0.235 & 0.179 & 0.159 & 0.147 & 0.124 & 0.112 & 0.0974 & 0.0866 & 0.0793 \\
\hline$\hat{\alpha}_{p}$ & 5.14 & 4.49 & 4.23 & 4.06 & 3.74 & 3.55 & 3.31 & 3.12 & 2.99 \\
\hline
\end{tabular}

Table 4: 80 days of age

Table 4: 80 days of age
\begin{tabular}{|c|ccccccccc|}
\hline$c_{P H}$ & $10^{-5}$ & $410^{-5}$ & $710^{-5}$ & $10^{-4}$ & $210^{-4}$ & $310^{-4}$ & $510^{-4}$ & $7.510^{-4}$ & $10^{-3}$ \\
\hline$\delta_{p}$ & 0.320 & 0.245 & 0.217 & 0.200 & 0.170 & 0.153 & 0.133 & 0.118 & 0.108 \\
\hline$\hat{\alpha}_{p}$ & 6.00 & 5.24 & 4.94 & 4.75 & 4.37 & 4.15 & 3.87 & 3.65 & 3.49 \\
\hline
\end{tabular}

It is worth pointing out that, in order to keep the length $\Gamma_{P}$ constant, considerable variations in the value of $c_{P H}$ can be compensated by means of comparatively small changes in the model parameters being considered. 
We remark that Figures 5, 6 are important in the framework of our work, whose aim is not just to propose a mathematical model for the dynamics of the growth plate, but also to understand how critical the role of the threshold $c_{P H}$ is, since reliable quantitative information on it is actually scarce. Such figures show that the regulatory loop is not very sensitive to $c_{P H}$ in a considerably large range, in the sense that if for instance $c_{P H}$ varies by a factor of 2 in the range $0.0004 \div 0.008$ the adjustment required on $\delta_{p}, \hat{\alpha}_{p}$ to achieve the same value of $\Gamma_{p}$ is less than $10 \%$.

Finally, note that both parameters $\delta_{p}$ and $\hat{\alpha}_{p}$, depends only of the diffusivity $D_{p}$. From (3.5) and (2.18) we can write

$$
\begin{aligned}
& \delta_{p}=\frac{D_{p}}{\Gamma_{p}^{2}} \Omega_{p}^{2} \\
& \hat{\alpha}_{p}=\frac{D_{p}}{\Gamma_{p} \gamma_{i}}\left(\Omega_{i} \Omega_{p} \sinh \Omega_{i} \sinh \Omega_{p}\right)=\frac{D_{p} c_{P H}}{\Gamma_{p} \hat{c}_{i}\left(\Gamma_{p}\right)} \Omega_{p} \sinh \Omega_{p},
\end{aligned}
$$

where $\Omega_{p}$ is determined by the equation

$$
\cosh \Omega_{p}=\frac{\hat{c}_{p}\left(\Gamma_{p}\right)}{c_{P H}} .
$$

Thus, a different choice for $D_{p}$ implies different values for the parameters $\delta_{p}$ and $\hat{\alpha}_{p}$ (both parameters are multiplied by $\frac{D_{p}}{D}$ ). Nevertheless the form of the graphics in figures 5 and 6 , and our main conclusions here, namely, the little sensitivity of the model to the precise value of $c_{P H}$ are independent of the value of $D_{p}$.

\section{Discussion and concluding remarks}

This work is concerned with a relevant aspect of primary ossification in rat tibia, a characteristic example of long bone formation in vertebrates. More precisely, a mathematical model for the interaction of two key signalling molecules, Ihh and PTHrP, has been presented and discussed. While other substances as Bone Morphogenetic Proteins, BMPs ([21]) or Vascular Endothelial Growth Factor, VGEF ([26]) have been reported as playing various roles in ossification, a consensus remains concerning the fundamental importance of the Ihh/PTHrP feedback loop in the process under consideration ([8], [19], [20], [21]). We have examined in detail the way in which Ihh and PTHrP act on chondrocytes in the Proliferative Zone (PZ). This is a part of the Growth Plate, the moving region where the transition from an advancing bone front to an invaded cartilage template takes place.

Our study is based on current knowledge about the manner in which the Ihh/PTHrP feedback loop takes place ([8], [19], [20],[21]). More precisely, we assume that Ihh stimulates the production of PTHrP at the Resting Zone, RZ. From there, a decreasing PTHrP gradient is established, that keeps chondrocytes 
at a proliferating stage (thus determining the length $\Gamma_{P}$ of PZ), until PTHrP concentration falls below a threshold value denoted by $c_{P H}$. The position $(z=0$ in our current notation) where this value is attained marks the transition to the Hyperthrophic Zone, HZ. To keep the feedback loop operating, cells at a thin region near $z=0$ produce a flux of Ihh. This last gives raise to a gradient of Ihh, which reaches up to the RZ, and regulates the production of PTHrP there, thus closing the loop.

In this article, an attempt has been made to present a mathematical model for the Ihh/PTHrP loop as simple as possible, so as to allow for explicit resolution in terms of the parameters involved. It should be considered that the Growth Plate of postnatal animals is a highly complex three-dimensional structure, whose experimental study presents many technical difficulties. As a result, experimental parameter determination is quite difficult, and in many cases a considerable degree of uncertainty remains about the accuracy of the corresponding measurements. For instance, in [14] estimates on the Ihh and PTHrP diffusivities were indirectly obtained by using bovine albumin fragments having similar molecular weight to that of Ihh or PTHrP. Although this is a widely used methodology, the question remains to ascertain whether the interaction of Ihh/PTHrP with the extracellular matrix (ECM), or with other ligands, might result in different effective diffusivities in the actual in vivo process. This makes all the more remarkable the fact, that we have already stressed, that the regulatory properties described by formulae (3.4) and (3.13) are independent of the particular choice of Ihh and PTHrP diffusivities made. This remark on the limits of experimental measurement also applies to the values reported in (2.20). In particular, a sharp difference appears there among the scale in which most constants are estimated $(\mu M)$ and that in which $c_{P H}$ is presented $(n M)$. Concerning the latter, measuring in vivo so small a value for a concentration at a particular location can only be accepted, we believe, after allowing for a wide fluctuation range. As a consequence, it seems that experimental estimates of that particular parameter have to be understood up to considerable fluctuations, even by one (or more) orders of magnitude.

A main goal of our work has been to obtain a mathematical model involving a reduced number of parameters, whose values are either experimentally derived in this work or alternatively could be found in the literature, and to analyze the dependence of solutions on changes in parameter values. In our view, uncertainty about parameter validation is a bonus to look for simple models. For instance, consideration of nonlinear kinetics (as those of Michaelis-Menten or Hill types) will lead to inclusion of further parameters that should then be estimated somehow. Needless to say, simplicity might result acceptable as far as conclusions derived from the model are consistent with, or represent a (motivated) challenge to, our current knowledge about the functioning of the Ihh/PTHrP loop. Bearing this statement in mind, we briefly recall below some of the results derived from the analysis of our model.

To begin with, we have considered the question of the efficiency of the Ihh/PTHrP loop to regulate the size of the Growth Plate. A way to address 
such issue consists in describing how perturbations in that loop affect to the length of the Proliferative Zone. We have addressed this issue in various manners. First, we have discussed in Section 3 how the length of $\mathrm{PZ}$ changes under small variations in the model data, which are likely to occur, even in physiological circumstances. In particular, one of the main conclusions of this work is that small changes in the flux parameters related to Ihh and PThrP production at the signalling interfaces RZ/PZ and PZ/HZ are efficiently damped out, since they induce modifications on the PZ length that are at least one order of magnitude lower than the perturbations (see (3.4)).

On the other hand, we have shown that, if Ihh concentration (respectively, PTHrP concentration) undergoes small variations at both signalling interfaces (respectively, at the RZ/PZ interface), the length of PZ itself experiences small changes, which are roughly proportional to those experienced by the concentrations. Another important conclusion of this work is that, as shown in (3.13) the length of $\mathrm{PZ}$ is rather insensitive to small fluctuations in the Ihh concentration at the $\mathrm{PZ} / \mathrm{HZ}$ interface. Moreover, as the same formula shows, a (small) increase in Ihh concentration at the RZ/PZ interface tends to reduce the length of PZ, whereas an increase of PTHrP there has the opposite effect. Note that such results hint at the robustness of the model under consideration. As has been already stressed, such conclusions are independent of the particular diffusivity coefficients taken for Ihh and PTHrP. It is worth remarking that such predictions seem to be in agreement with regulation properties of the Growth Plate obtained form experimental studies (cf. for instance [20], [8]). In particular, it has been observed in [20] that misexpression of Thh prevents proliferating chondrocytes to enter into the hypertrophic phase, and that markers of hypertrophic cartilage are suppressed by Ihh. Furthermore, targeted disruption of the PTHrP gene in mice has an effect opposite to that of overexpressing Ihh. More precisely, in PTHrP-deficient mice, the transition from the proliferative to the hypertrophic zone is accelerated, which leads to a premature ossification. All these findings are compatible with our results above. It should be noted in this respect that when Ihh concentration is increased at the RZ/PZ interface, but the concentration of PTHrP is not modified there, the flux condition (2.5) implies a steeper slope for the PTHrP profile, thereby shortening the length of PZ. In fact (3.13) provides an estimate on the relative small perturbations of both signalling cues to allow for enlarging of the PZ.

A second way to obtain insight into the regulatory properties implicit in our model consists in estimating how modifications, not necessarily small, in the model parameters should be counterbalanced in order to keep the length of PZ constant. We have addressed such question in a particular case, and we have shown that even variations of up to two orders of magnitude in the value of $c_{P H}$ can be accommodated by means of rather small changes in some of the model parameters in order to keep $\Gamma_{P}$ constant. This strongly suggests that our model is rather insensitive to changes in the value of $c_{P H}$, which is by far the parameter less accessible to experimental measurement.

At this juncture, it is worth remarking on how our results are related to previous modelling work in the field. The approach in this article is similar to that 
in references [9] and [10] (see also [11] and [13]). In particular, in [9] three signalling molecules (Ihh, PTHrP and VGEF) were considered, although only the first two actually were assumed to operate at the PZ. A time-dependent, nonlinear reaction-diffusion model was considered in [9] and numerically simulated there. In that model, molecule synthesis (described by means of a Hill-type dynamics) plays an important role. Since some of the parameters therein involved remain unknown, they had to be externally fitted from what was referred to as a stable Growth Plate model. In particular, it was observed that increased (respectively, decreased) PTHrP synthesis rates result in longer (respectively, shorter) PZ. No significant change was observed in the HZ length. A similar result was obtained concerning Ihh synthesis rates. A related approach was then pursued in [10], where this time only equilibrium conditions were considered, as in our current work here. Moreover, the Ihh/PTHrP concentration profiles in the PZ were assumed to be strictly linear. Again, some of the parameters in the model remained unknown (although useful references for some of them, including our $c_{P H}$, were provided). In this way, a number of relevant hints about the way in which PZ is regulated were discussed. Concerning HZ, uncertainty remains about the validity of the proposed behaviour, since some of the estimates provided in [10] seem to yield unrealistic values. We point out however that our work, which has considerably benefited from [9] and [10], can be considered as a further step in that modelling direction. In particular the mathematical formalism has been further simplified, as to allow for an explicit solution instead of a numerical one (which, as seen in [9], [10] often shows what seem to be spurious oscillations).

We conclude this Section by observing that, however important they are for endochondral ossification, Ihh and PTHrP are merely two, out of a larger set, of signalling molecules, and in order to gain further insight into the dynamics of the Growth Plate, more complex models have surely to be considered. These should account for more sophisticated biochemical circuitry (that could possibly incorporate the Ihh/PTHrP loop in a modular way), but also for a detailed analysis of the mechanical aspects of Growth Plate regulation. In particular, it would be quite interesting to check the validity of our results for the cases considered, for instance, in [11] and [13]. We intend to address these issues in future work.

Acknowledgements: This work has been partially supported by MICINN Research Grant MTM2008-01867, FIS2008-00200. The authors are grateful to two anonymous referees for a number of suggestions which have led to an improvement in the presentation of our results.

\section{References}

[1] Hunziker E.B.(1994). Mechanism of longitudinal bone growth and its regulation by growth plate chondrocyte. Micros. Res. Tech. 28, 505-519. 
[2] Rivas R. and Shapiro F. (2002). Structural stages in the development of the long bones and epiphysis. J. Bone Joint Surg. 84 (1), 85-100.

[3] Kronenberg H.M.(2003). Developmental regulation of the growth plate. Nature $423,332-336$.

[4] Herrero M.A. and Lopez J.M. (2005). Bone formation: biological aspects and modelling problems. Journal of Theoretical Medicine (now Computational and Mathematical Methods in Medicine) 6 (1) 41-55.

[5] Mackie E.J., Ahmed Y.A., Tatarczuch L., Chen K. S., and Mirams M. (2008) Endochondral ossification: How cartilage is converted into bone in the developing skeleton. The International Journal of Biochemistry \& Cell Biology 40, 46-62.

[6] Nilsson O., Marino R., De Luca F., Phillip M., Baron J. (2005). Endocrine regulation of the growth plate. Horm Res. 64, 157-65.

[7] Shimo T., Gentili C., Iwamoto M., Wu C., Koyama E. and Pacifici M. (2004). Indian hedgehog and syndecans-3 coregulate chondrocyte proliferation and function during chick limb skeletogenesis. Dev. Dyn. 229, 607-617.

[8] Weir E.C., Philbrick W.M., Amling M, Neff L.N., Baron R. and Broadus A.E. (1996). Targeted overexpression of parathyroid hormone-related peptide in chondrocytes causes chondrodysplasia and delayed endochondral bone formation. Proc. Natl. Acad. Sci. USA 93, 10240-10245.

[9] Brouwers J.E.M., van Donkelaar C.C., Sengers B.G. and Huiskes R. (2006). Can the growth factors PTHrP, Ihh and VGEF, together regulate the development of a long bone?. Journal of Biomechanics 39, 2774-2782.

[10] Van Donkelaar C.C. and Huiskes R. (2007). The PTHrP-Ihh fedback loop in the embryonic growth plate allows PTHrP to control hypertrophy and Ihh to regulate proliferation. Biomechan. Model Mechanobiol. 6, 55-62.

[11] Garzón-Alvarado G.A, García-Aznar J.M., and Doblaré M. (2009). A reaction-diffusion model for long bones growth. Biomech. Model. Mechanobiol. 8, 381-395.

[12] Moreo P., García-Aznar J.M., and Doblaré M. (2009). Bone ingrowth on the surface of endosseous implants. Part 1: Mathematical model. J. Theor. Biol. 260, 1-12.

[13] Garzón-Alvarado G.A, García-Aznar J.M., and Doblaré M. (2009). Appearance and location of secondary ossification centres may be explained by a reaction-diffusion mechanism. Comp. In Biology and Medicine 39, 554-561.

[14] Williams R.M., Zipfel W.R., Tinsley M.L. and Farnum C.E. (2007). Solute Transport in Growth Plate Cartilage: In Vitro and In Vivo. Biophysical Journal 93, 1039-1050. 
[15] Kalderon D (2002). Similarities between the Hedgehog and Wnt signalling pathways. Trends Cell Biol. 12; 523-531.

[16] Saha D., and Schaffer D.(2006). Signal dynamics in Sonic Hedgehog tissue patterning. Development 133; 889-900.

[17] Edwards R.C., Ratcliffe W.A., Walls J., Morrison J.M., Ratcliffe J.G., Holder R. and Bundred N.J. (1995). Parathyroid hormone-related protein $(\mathrm{PTHrP})$ in breast cancer and in benign breast tissue. European J. Cancer 31,$3 ; 334-339$.

[18] Meinhardt H. (1982). Models of biological pattern formation. Academic Press.

[19] Kobayashi T. Chung, U.I., Schipani E., Starbuck M., Karsenty G., Katagiri T. Goad D.L., Lanske B. and Kronenberg H.M. (2002). PTHrP and Indian hedgehog control differentiation of growth plate chondrocytes at multiple steps. Development 129, 2977-2986.

[20] Vortkamp A., Lee K., Lanske B., Segre G.V., Kronenberg H.M. and Tabin C.J. (1996). Regulation of rate of cartilage differentiation by Indian Hedgehog and PTH-related protein. Science 273, 613- 622.

[21] Minina E., Wenzel H.M., Kreschel C., Karp S., Gaffield W., McMahon A. and Wortkamp A. (2001). Bmp AND Ihh/PTHrP signalling interact to coordinate chondrocyte proliferation and differentiation. Development 128 , 4523-4534.

[22] Zhao Q., Brauer P.R., Xiao L. Mc Guire M.H. and Yee J.A. (2002). Expression of parathyroid hormone -related peptide (PthrP) and its receptor (PTH1R) during the histogenesis of cartilage and bone in the chicken mandibular process. J. Anat. 201, 137-151.

[23] Pathi S., Pagan-Westphal S., Baker D.P., Garber E.A. Rayhorn P., Bumcrot D. Tabin C.J. Blake Pepinsky R. and Williams K.P. (2001). Comparative biological responses to human Sonic, Indian and desert hedgehog. Mech. Dev. 106, 107-117.

[24] Cobo A., López J.M., Carbajo E, Santos F., Alvarez J., Fernández M., Weruaga A. (1999). Growth plate cartilage formation and resorption are differentially depressed in growth retarded uremic rats. J. Am. Soc. Nephrol. $10(5), 971-9$

[25] Alvarez J., Balbín M., Santos F., Fernández M., Ferrando S., and López J. M. (2000). Different bone growth rates are associated with changes in the expression patterns of types II and X collagens and collagenase 3 in proximal growth plates of the rat tibia. J. Bone Miner. Res. 15, 82-94. 
[26] Colnot C., Lu C., Hu D., and Helms J.A. (2004). Distinguishing the contributions of the perichondrium, cartilage and vascular endothelium to skeletal development. Developmental Biology 269, 55-69.

\section{Appendix A: Discarding convective effects}

We present below an argument that shows that convective effects can be safely neglected when compared with reaction-absorption ones in the model described in Section 2. To this end, we keep to the notation in subsection 2.2 and consider the more general form of equations (2.1)-(2.2) given by

$$
\begin{aligned}
& -D \frac{d^{2} \hat{c}_{i}}{d \hat{z}^{2}}+\frac{d}{d \hat{z}}\left(\hat{c}_{i} \hat{v}\right)+\delta_{i} \hat{c}_{i}=0 \quad \text { for } \quad 0<\hat{z}<\Gamma_{P}, \\
& -D \frac{d^{2} \hat{c}_{p}}{d \hat{z}^{2}}+\frac{d}{d \hat{z}}\left(\hat{c}_{p} \hat{v}\right)+\delta_{p} \hat{c}_{p}=0 \quad \text { for } \quad 0<\hat{z}<\Gamma_{P},
\end{aligned}
$$

which clearly reduce to $(2.1)-(2.2)$ when the convective velocity $\hat{v}$ vanishes. Note that $\hat{v}=\hat{v}(\hat{z})$ denotes the cell velocity in the chosen frame of reference so that

$$
\hat{v}(\hat{z})=\chi_{P}\left(\Gamma_{P}-\hat{z}\right),
$$

where $\chi_{P}$ is the cell proliferation rate within PZ. To proceed further, we make use of the following non-dimensional variables which can be considered as a generalization of $(2.8)$.

$$
\begin{aligned}
& z=\frac{\hat{z}}{\Gamma_{P}}, \quad c_{i}=\frac{\hat{c}_{i}}{c_{P H}}, \quad c_{p}=\frac{\hat{c}_{p}}{c_{P H}}, \quad \text { and } \\
& v(z)=v_{0}(1-z), \quad \text { where } v_{0}=\hat{v}(0)=\chi_{P} \Gamma_{P} .
\end{aligned}
$$

Using (A.4), (A.1) transforms into

$$
-\frac{D}{\Gamma_{P}^{2}} \frac{d^{2} c_{i}}{d z^{2}}+\frac{v_{0}}{\Gamma_{P}}\left((1-z) \frac{d c_{i}}{d z}-c_{i}\right)+\delta_{i} c_{i}=0 ; \quad 0<z<1 .
$$

We next make use of the diffusive time $\tau_{D}$ (defined in (2.10)) and the convective time $\tau_{C}$ defined below

$$
\tau_{C}=\frac{\Gamma_{P}}{v_{0}}, \quad \tau_{D}=\frac{\Gamma_{P}^{2}}{D} .
$$

On setting $\Omega_{i}^{2}=\delta_{i} \tau_{D}$ (cf. (2.9)) it follows from (A.6) that (A.5) can be rewritten as

$$
\frac{d^{2} c_{i}}{d z^{2}}-\frac{\tau_{D}}{\tau_{C}}\left((1-z) \frac{d c_{i}}{d z}-c_{i}\right)=\Omega_{i}^{2} c_{i}, \quad 0<z<1 .
$$

If we now take $D=50 \mu \mathrm{m}^{2} / \mathrm{s}$ (see Section 2) and $\Gamma_{P}$ as given in (2.25) we have

$$
\tau_{D}=648 s, 516 s, \text { and } 378 s,
$$


for 21, 35 and 80 days old animals, respectively. We now take advantage of the estimates for the proliferation rates provided in Table 5 below

TABLE 5

$\begin{array}{cccccc}\begin{array}{c}\text { LI. 1hr. } \\ (\%)\end{array} & \begin{array}{c}\text { LI. 3hr. } \\ (\%)\end{array} & \begin{array}{c}\text { difference } \\ (\%)\end{array} & \begin{array}{c}\text { Duplication } \\ \text { (percentage) }\end{array} & \begin{array}{c}\text { Duplication } \\ \text { time (hr.) } \\ \text { for marked } \\ \text { chondrocytes }\end{array} & \begin{array}{c}\text { Cell cycle } \\ \text { (in hours) }\end{array} \\ \begin{array}{c}\mathbf{2 1} \text { days } \\ 13.4\end{array} & \begin{array}{l}20.8 \\ \pm 3.9\end{array} & 7.4 & 55.2 & 3.6 & 27.0 \\ \pm 2.9 & & & & \\ \mathbf{3 5} \text { days } & & & & & \\ 16.3 & 25.9 & 9.6 & 58.9 & & \\ \pm 3.6 & \pm 5.1 & & & & \\ \mathbf{8 0} \text { days } & & & & & \\ 7.9 & 10.6 & 2.7 & 34.2 & & 74.9 \\ \pm 1.7 & \pm 2.2 & & & \end{array}$

The labelling index (LI) was calculated as the percentage of nuclear profiles which are labelled by means of BrdU marking techniques. Differences between LI at 1 and 3 hours were used to estimate the time needed to duplicate the total number of proliferating chondrocytes (cell cycle time).

By Table 5 we have that

$$
\begin{aligned}
& \chi_{P}=\frac{1}{20.8 \cdot 3600} s^{-1}=1.34 \cdot 10^{-5} s^{-1}, \quad \text { for } 21 \text { days old rats, } \\
& \chi_{P}=\frac{1}{25.9 \cdot 3600} s^{-1}=1.07 \cdot 10^{-5} s^{-1}, \quad \text { for } 35 \text { days old rats, } \\
& \chi_{P}=\frac{1}{10.6 \cdot 3600} s^{-1}=2.62 \cdot 10^{-5} s^{-1}, \quad \text { for } 80 \text { days old rats, }
\end{aligned}
$$

whence

$$
\tau_{C}=\frac{\Gamma_{P}}{v_{0}}=\frac{1}{\chi_{P}}=7.5 \cdot 10^{4} \mathrm{~s}, 9.3 \cdot 10^{4} \mathrm{~s} \text { and } 3.8 \cdot 10^{4} \mathrm{~s} .
$$

It then turns out that

$$
0<\frac{\tau_{D}}{\tau_{C}}=0.0086,0.0055,0.01 \ll 1,
$$

and the second term in the left of (A.7) results to be much smaller than the remaining ones, which are of size $\mathcal{O}(1)$ by the rescaling made in (A.4). The other age groups are dealt with similarly.

If we take different diffusivities $D_{i}$ and $D_{p}$ in equations (A.1) and (A.2) it is easily checked that the above quotients are multiplied by $\frac{D}{D_{i}}$ and $\frac{D}{D_{p}}$ for the equations for $c_{i}$ and $c_{p}$ respectively. It the follows that, if $D_{i}$ and $D_{p}$ are smaller than $D$, convective effects remain negligible whenever such diffusivities are up to one order of magnitude smaller than $D$. Convective effects can always be discarded for values of $D_{i}$ and $D_{p}$ larger than $D$. 